\title{
Heterogeneous Sticky-Information Economies*
}

\author{
Carlos Carvalho ${ }^{* *}$
}

\begin{abstract}
The literature that emerged from Mankiw and Reis' (2002) proposal of sticky information as an alternative to sticky-price models has focused on economies populated with (ex-ante) identical firms. This paper analyzes the implications of heterogeneity in the degree of information stickiness among price-setting firms in different sectors for the dynamic response of the economy to various shocks. I compare multisector sticky-information models with otherwise identical one-sector economies that feature the same frequency of information updating. I find that the effects of such shocks on the output gap - the difference between actual output and the output level that would prevail in the absence of information frictions - are larger in heterogeneous economies.
\end{abstract}

Keywords: Sticky-information; Heterogeneity.

JEL codes: E3.

Submitted on $11 / 13 / 2015$. Revised on $7 / 17 / 2017$.

This paper is based on chapter 3 of my Ph.D. dissertation, and was never submitted for publication. I would like to thank David Romer, Ricardo Reis, Vasco Cúrdia, and participants from ESWC 2005, LAMES 2004, EEA Meeting 2004, Student Macro/International Workshop at Princeton University, and EPRU seminar at the University of Copenhagen for helpful comments. Any remaining errors are my own. The views expressed in this paper do not necessarily reflect the position of the Central Bank of Brazil.

** Banco Central do Brasil and PUC-Rio. Email: cvianac@econ.puc-rio.br. 


\section{Introduction}

As with most work on sticky prices, the literature that emerged after Mankiw and Reis (2002) proposed sticky information as an alternative price-setting model has focused on economies with (ex-ante) identical firms. While there is hardly any direct evidence on the frequency with which firms update their information in order to set prices, there is no a priori reason why firms in different sectors should behave similarly in this dimension. In fact, sticky-information models are best seen as a reduced form of a microfounded model in which firms face explicit costs to acquire and process information, and optimally choose for how long to wait before updating their information again (Reis, 2006). When microfounded, the optimal length of inatentiveness depends on primitives such as the size of information costs, the importance of sectoral shocks to demand and marginal costs, and the shape of the profit function. Because these primitives are likely to differ across sectors or industries, we should actually expect firms in different sectors to update information at different frequencies.

This paper studies the implications of heterogeneity in the degree of information stickiness for aggregate dynamics, focusing on how it affects the response of the output gap to various kinds of shocks. The output gap here is the difference between actual and the "natural" level of output, which is the level that would prevail if all firms adjusted prices continuously and with full information. Actual and natural output levels differ due to information frictions. I emphasize informational frictions of the kind advocated by Mankiw and Reis (2002), whereby firms adjust prices all the time, but only update their knowledge of the state of the economy sporadically.

For a given heterogeneous sticky-information economy, I isolate the effects of heterogeneity by comparing how it affects the response of the output gap to various types of shocks, relative to an otherwise identical economy with homogenous pricing behavior and the same average frequency of information updating. In particular, I study multisector versions of the original sticky-information model of Mankiw and Reis (2002), and of the variation analyzed by Dupor and Tsuruga (2005), which replaces the random intervals between information updating dates with spells of inatentiveness with fixed duration. 
When compared with one-sector economies with the same average frequency of information updating, heterogeneous sticky-information economies display larger and more persistent responses to a variety of shocks. To provide an intuitive metric for the extent to which heterogeneity plays the role of a propagation mechanism for the various shocks, I follow Carvalho (2006) and ask how the calibration of the average frequency of information updating in an identical-firms economy needs to be "distorted" to approximate the dynamics of the heterogeneous economy. For calibrated versions of the two aforementioned sticky-information models, I find the average frequency of information updating in the identical-firms economy that minimizes the distance of its impulse response functions to those of the heterogeneous economy, with all other parameters held fixed. I also study a related problem, in which the degree of real rigidities in the identical firms economy is also used to obtain a better fit. The common lesson that emerges from the results is clear: Although identical-firms models might be able to provide a reasonable description of a more complex, heterogeneous sticky-information economy, this typically requires parameter values which seem unrealistic if interpreted literally.

The rest of the paper is organized as follows. Subsection 2 presents the basic setup and introduces the two price-setting models subsequently. Subsection 3 presents the analytical results. Quantitative results follow in Subsection 4. The last subsection concludes. All proofs are in the Appendix.

\section{Models}

I start with the description of the underlying economic environment that is common to the two sticky-information models. For that purpose, it is useful to present the frictionless economy that would obtain in the absence of any impediments to continuous information updating. The frictions that give rise to the two stickyinformation models are introduced in specific subsections. For convenience, I work in continuous time, and avoid the technical complications associated with continuoustime uncertainty by making use of approximate versions of the models in which certainty-equivalence holds, and then focusing on the effects of one-time shocks. 


\subsection{Frictionless economy}

A representative consumer derives utility from a Dixit-Stiglitz composite of differentiated consumption goods and supplies a continuum of differentiated types of labor to monopolistically competitive firms, which he owns. The latter are divided into sectors, and indexed by their sector, $k \in K$, and by $j \in[0,1]$. The distribution of firms across sectors is summarized by a density function $f$ on $K$ (with cdf $F$ ). Each firm hires labor of a specific type in a competitive market to produce a likewise specific variety of the consumption good with a technology subject to shocks. I work with a cashless-limit economy, as in Woodford (2003). In the frictionless environment described in this subsection firms have full information about the state of the economy at all times. As a result, there are no differences between the various sectors, and the notation is introduced here for convenience. In subsequent subsections, the extent of informational frictions is allowed to vary across sectors, leading to a meaningful such distinction.

The representative consumer maximizes:

$$
E_{0} \int_{0}^{\infty} e^{-\rho t}\left(\frac{C(t)^{1-\sigma}-1}{1-\sigma}-V(t) \int_{k \in K} f(k) \int_{0}^{1} \frac{L_{k j}(t)^{1+\frac{1}{\varphi}}}{1+\frac{1}{\varphi}} d j d k\right) d t
$$

$$
\begin{aligned}
\text { s.t. } B(t)=B(0)+\int_{0}^{t} \int_{k \in K} f( & (k) \\
& \int_{0}^{1} L_{k j}(s) W_{k j}(s) d j d k d s-\int_{0}^{t} P(s) C(s) d s \\
& +\int_{0}^{t} \Omega(s) d Q(s)+\int_{0}^{t} \Omega(s) d D(s), \text { for } t \geq 0
\end{aligned}
$$

where $\rho$ is the discount rate, $C(t)$ is consumption of the composite good, $L_{k j}(t)$ is the supplied quantity of type $k j$ labor, $W_{k j}(t)$ is the associated nominal wage, $V(t)$ is a shock to the disutility of labor, $B(t)$ denotes total financial wealth, $P(t)$ is a price index to be specified below, $Q(t)$ is the collection of asset prices, $D(t)$ is the collection of corresponding cumulative dividend processes, and $\Omega(t)$ is the trading strategy, assumed to satisfy conditions that preclude Ponzi schemes. The parameters $\sigma^{-1}$ and $\varphi$ stand for the intertemporal elasticity of substitution in consumption and the (Frisch) elasticity of labor supply, respectively. 
The composite consumption good is given by:

$$
\begin{aligned}
C(t) & \equiv\left[\int_{k \in K} f(k)^{\frac{1}{\eta}} C_{k}(t)^{\frac{\eta-1}{\eta}} d k\right]^{\frac{\eta}{\eta-1}}, \\
C_{k}(t) & \equiv f(k)\left[\int_{0}^{1} C_{k j}(t)^{\frac{\varepsilon-1}{\varepsilon}} d j\right]^{\frac{\varepsilon}{\varepsilon-1}}
\end{aligned}
$$

where $C_{k}(t)$ is the subcomposite of goods produced by firms in sector $k$, and $C_{k j}(t)$ is consumption of the variety of the good produced by firm $j$ from sector $k$ (henceforth "firm $k j$ "). The elasticity of substitution between varieties within a sector is $\varepsilon>1$, and $\eta>0$ is the elasticity of substitution across different sectors. Denoting by $P_{k j}(t)$ the price charged by firm $k j$ at time $t$, the underlying consumption price index is:

$$
P(t)=\left[\int_{k \in K} f(k) P_{k}(t)^{1-\eta} d k\right]^{\frac{1}{1-\eta}},
$$

where $P_{k}(t)=\left[\int_{0}^{1} P_{k j}(t)^{1-\varepsilon} d j\right]^{\frac{1}{1-\varepsilon}}$ is a sectoral price index.

In later subsections I work with log-linear approximate models in which certainty-equivalence holds, and moreover make assumptions that preclude having to solve the optimal-control problem associated with consumption-savings decisions under uncertainty in continuous time. Therefore, the subset of optimality conditions for the consumer's problem that are used later is:

$$
\begin{aligned}
\frac{W_{k j}(t)}{P(t)} & =V(t) \frac{L_{k j}(t)^{\frac{1}{\varphi}}}{C(t)^{-\sigma}}, k j \in K \times[0,1], \\
C_{k}(t) & =f(k) C(t)\left(\frac{P_{k}(t)}{P(t)}\right)^{-\eta}, k \in K, \\
C_{k j}(t) & =f(k)^{-1} C_{k}(t)\left(\frac{P_{k j}(t)}{P_{k}(t)}\right)^{-\varepsilon}, k j \in K \times[0,1],
\end{aligned}
$$

In this frictionless environment, firms solve a simple, static profit maximization problem. Firm $k j$ hires labor of its specific type in a competitive labor market to produce its variety of the consumption good according to a linear technology subject to a productivity shock:

$$
Y_{k j}(t)=A(t) N_{k j}(t),
$$


where $Y_{k j}(t)$ is the production of its variety, $N_{k j}(t)$ is the specific labor input, and $A(t)$ is the productivity shock. The firm's problem is then given by:

$$
\begin{aligned}
& \max _{P_{k j}^{*}(t)}\left[P_{k j}^{*}(t) Y_{k j}(t)-W_{k j}(t) N_{k j}(t)\right] \\
& \text { s.t. } Y_{k j}(t)=A(t) N_{k j}(t), \\
& \qquad Y_{k j}(t)=\left(\frac{P_{k j}^{*}(t)}{P_{k}(t)}\right)^{-\varepsilon}\left(\frac{P_{k}(t)}{P(t)}\right)^{-\eta} Y^{n}(t),
\end{aligned}
$$

where

$$
\begin{aligned}
Y^{n}(t) & \equiv\left[\int_{k \in K} f(k)^{\frac{1}{\eta}} Y_{k}(t)^{\frac{\eta-1}{\eta}} d k\right]^{\frac{\eta}{\eta-1}}, \\
Y_{k}(t) & \equiv f(k)\left[\int_{0}^{1} Y_{k j}(t)^{\frac{\varepsilon-1}{\varepsilon}} d j\right]^{\frac{\varepsilon}{\varepsilon-1}},
\end{aligned}
$$

and where I have used the market clearing conditions in the goods markets. The optimal frictionless price is given by the usual mark-up rule:

$$
P_{k j}^{*}(t)=\frac{\varepsilon}{\varepsilon-1} \frac{W_{k j}(t)}{A(t)} .
$$

The model is closed with a monetary policy specification that ensures existence and uniqueness of a rational expectations equilibrium. Given that monetary disturbances do not affect real variables in this frictionless environment, in equilibrium output evolves stochastically only as a function of the productivity and labor supply shocks. Following Woodford (2003), this level of output in the frictionless economy, denoted $Y^{n}(t)$, is what I term the natural output level:

$$
Y^{n}(t)=\left(\frac{\varepsilon-1}{\varepsilon}\right)^{\frac{\varphi}{1+\sigma \varphi}} \frac{A(t)^{\frac{1+\varphi}{1+\sigma \varphi}}}{V(t)^{\frac{\varphi}{1+\sigma \varphi}}} .
$$

The next two subsections introduce the different sticky-information models, which arise due to different assumptions about how firms update their knowledge of the state of the economy. To highlight the effects of heterogeneity in price setting, sectors only differ in the degree of information stickiness, and hence they are uniquely identified by this dimension. 


\subsection{Sticky information as in Mankiw and Reis (2002)}

The first model generalizes the original paper of Mankiw and Reis (2002) to a setting with heterogeneity in information frictions. Firms update their information sets at a constant rate, which differs across sectors. The incidence of these "updating events" is independent across firms. At each instant, firms set prices to maximize current expected profits, conditional on their current (possibly outdated) information.

At time $t$, a firm $k j$ that last updated its information set at time $t-s$ solves: $^{1}$

$$
\max _{X_{k j}^{M R}(t-s, t)} E_{t-s}\left[\left(X_{k j}^{M R}(t-s, t)-\frac{W_{k j}^{M R}(t)}{A(t)}\right)\left(\frac{X_{k j}^{M R}(t-s, t)}{P_{k}^{M R}(t)}\right)^{-\varepsilon}\left(\frac{P_{k}^{M R}(t)}{P^{M R}(t)}\right)^{-\eta} Y^{M R}(t)\right],
$$

where the " $M R$ " superscript stands for "Mankiw and Reis." Thus, it sets:

$$
X_{k j}^{M R}(t-s, t)=\frac{\varepsilon}{\varepsilon-1} \frac{E_{t-s}\left[P_{k}^{M R}(t)^{\varepsilon-\eta} P^{M R}(t)^{\eta} Y^{M R}(t) W_{k j}^{M R}(t) A(t)^{-1}\right]}{E_{t-s}\left[P_{k}^{M R}(t)^{\varepsilon-\eta} P^{M R}(t)^{\eta} Y^{M R}(t)\right]} .
$$

Firms from sector $k$ that share the same information set (from $s \leq t$, say) charge the same price, denoted $X_{k}^{M R}(s, t)$. Letting $\alpha_{k}>0$ denote the (constant) hazard rate of information updating for a firm in sector $k$, sectoral price indices can be written as:

$$
P_{k}^{M R}(t)=\left[\int_{-\infty}^{t} \alpha_{k} e^{-\alpha_{k}(t-s)} X_{k}^{M R}(s, t)^{1-\varepsilon} d s\right]^{\frac{1}{1-\varepsilon}}
$$

\subsection{Fixed-duration sticky information}

This subsection introduces a generalized version of the fixed-duration, staggered sticky-information model of Dupor and Tsuruga (2005). Firms from sector $k$ update their information sets at intervals of constant length $d_{k}$. Information updating dates within each sector are uniformly staggered across time.

Again, firms are not prevented from changing prices continuously, and so they set prices exactly as in the previous subsection. To differentiate variables in the

1 As in Mankiw and Reis (2006, 2007), firms with outdated information do not make any inference about the state of the economy in non-information-updating times. 
two sticky-information economies, we now use a " $F D$ " superscript, which stands for "fixed duration." Sectoral price levels are then given by:

$$
P_{k}^{F D}(t)=\left[\int_{t-d_{k}}^{t} \frac{1}{d_{k}} X_{k}^{F D}(s, t)^{1-\varepsilon} d s\right]^{\frac{1}{1-\varepsilon}}
$$

\subsection{Counterfactual one-sector economies}

The effects of heterogeneity in each economy will be measured through a comparison with a counterfactual one-sector economy that has the same average frequency of information updating, and is otherwise identical to the underlying heterogeneous economy. Thus, the one-sector MR sticky-information economy has firms updating their information sets at a rate given by $\bar{\alpha}=\int_{k \in K} f(k) \alpha_{k} d k$. In turn, the one-sector fixed-duration sticky-information model has firms updating their knowledge of the underlying state of the economy at intervals of length $\bar{d}=\left(\int_{k \in K} f(k) d_{k}^{-1} d k\right)^{-1} .^{2}$

\section{The effects of shocks - analytical results}

I solve the models by log-linearizing around a deterministic steady state, and then consider the dynamic response of the output gap to the various shocks. ${ }^{3}$ I start by providing analytical results under some simplifying assumptions. I leave the specification of monetary policy implicit by postulating that nominal aggregate demand is exogenous. This is a common assumption in the Monetary Economics literature (e.g., Mankiw and Reis 2002) that is helpful to highlight the features of the "supply side" of the model. I also impose restrictions on parameters so as to rule out strategic complementarity or substitutability in pricing decisions. For ease of presentation, I analyze the effects for each type of shock in the two models. Since certainty equivalence holds in the log-linear models, I focus on the dynamic response of the economies to one-time shocks. Finally, I look at a scalar measure of the effects of the shocks, given by the discounted cumulative effect on the output gap.

2 This formula follows from the fact that the frequency of updating associated with a fixed duration spell of $d_{k}$ is given by $d_{k}^{-1}$.

3 Since there are no impediments to continuous price adjustment, allowing for non-zero inflation is straightforward. 


\subsection{Common simplifying assumptions}

In the log-linear versions of the two economies, firms from sector $k$ that last updated information at $t-s$ set: $^{4}$

$$
x_{k}^{J}(t-s, t)=E_{t-s}\left[\frac{\varphi^{-1}+\sigma}{1+\varphi^{-1} \varepsilon} y^{J}(t)+\frac{1+\varphi^{-1} \eta}{1+\varphi^{-1} \varepsilon} p^{J}(t)+\frac{\varphi^{-1}(\varepsilon-\eta)}{1+\varphi^{-1} \varepsilon} p_{k}^{J}(t)+\frac{v(t)-\left(1+\varphi^{-1}\right) a(t)}{1+\varphi^{-1} \varepsilon}\right],
$$

where $J=$ " $M R$ ", " $F D$ ". In both cases, the log-linear natural output level evolves according to:

$$
y^{n}(t)=\frac{1+\varphi}{1+\sigma \varphi} a(t)-\frac{\varphi}{1+\sigma \varphi} v(t) .
$$

Therefore, (5) can be rewritten as:

$$
x_{k}^{J}(t-s, t)=E_{t-s}\left[\frac{1+\varphi^{-1} \eta}{1+\varphi^{-1} \varepsilon} p^{J}(t)+\frac{\varphi^{-1}(\varepsilon-\eta)}{1+\varphi^{-1} \varepsilon} p_{k}^{J}(t)+\frac{\varphi^{-1}+\sigma}{1+\varphi^{-1} \varepsilon}\left(y^{J}(t)-y^{n}(t)\right)\right] .
$$

When $\frac{\varphi^{-1}(\varepsilon-\eta)}{1+\varphi^{-1} \varepsilon}=0, \theta \equiv \frac{\varphi^{-1}+\sigma}{1+\varphi^{-1} \varepsilon}$ corresponds to the Ball and Romer (1990) index of real rigidities. In that case, prices are strategic complements (substitutes) if $\theta<1$ $(>1)$. Parameterizations that lead to $\theta=1$ imply neither complementarity nor substitutability, and I refer to them as involving strategic neutrality in price setting. Such parameterizations arise, for instance, under logarithmic consumption utility and linear disutility of labor.

Denoting (log) nominal aggregate demand (or nominal income) by $m(t) \equiv y(t)+$ $p(t)$, under strategic neutrality the equation for optimal price setting simplifies to:

$$
x_{k}^{J}(t-s, t)=E_{t-s}\left[m(t)-y^{n}(t)\right] .
$$

That is, positive surprises to nominal income and negative surprises to the natural rate of output have the same effect on pricing decisions. As a result, the effects of any shock on the output gap - be it a nominal income, productivity, or labor supply

4 From now on, lowercase variables denote log-deviations from the steady state. 
shock - can be analyzed with just one of those cases. ${ }^{5}$ For concreteness, I focus on nominal aggregate demand shocks, which implies $y^{n}(t)=0$ at all times.

In each case, I start from the steady state and assume that, without loss of generality, the shock hits at time zero. I focus on the effects of the shock on the output gap, and for that purpose I define its normalized and discounted cumulative effect as:

$$
\Gamma^{J}=\frac{1}{\text { size of shock }} \int_{0}^{\infty} e^{-\rho t}\left(y^{J}(t)-y^{n}(t)\right) d t .
$$

Finally, for convenience I assume that sectors are effectively identified with their (expected) duration of price plans, in the sense that the density $f$ is actually defined over such expected durations. Moreover, I assume that there is a sector for each possible (expected) duration between zero and $\sup _{k \in K}\left\{d_{k}\right\}$. This allows me to write $f(\tau)$ instead of $f\left(k \in K \mid d_{k}=\tau\right)$, which makes expressing and manipulating the mathematical results easier.

\section{$3.2 \quad$ Level shocks}

Starting from a zero-inflation steady state, at $t=0$ nominal aggregate demand is hit by a shock of size $\bar{m}$, which then decays exponentially at rate $\delta \geq 0$. The particular case of a permanent level shock (which corresponds to nominal income being a random walk) obtains when $\delta=0$. For $t \geq 0$, the path for nominal aggregate demand is therefore given by $m(t)=\bar{m} e^{-\rho t}$. To solve for the response of aggregate variables, the relevant distinction is between firms that already updated their pricing plans after the shock, and firms that are still setting prices based on their previous information.

Under strategic neutrality in price setting, firms which get to update their information set:

$$
x_{k}^{J}(s, t)=E_{s}[m(t)]
$$

5 For this result to hold, it is crucial that nominal income be unaffected by the other shocks. This clearly imposes restrictions on the nature of the underlying monetary policy. If policy reacts to endogenous variables, in principle it will react, at least indirectly, to all disturbances. So, there might be specifications for monetary policy that invalidate the conclusions based on this restriction. However, it might still be the case that the qualitative results that I obtain under this restriction hold when monetary policy is described by an empirically plausible interest rate rule. This is a question that I leave for future research. 
for $J=$ " $M R$ ", " $F D ", 0 \leq s \leq t$. ${ }^{6}$ The details of aggregation, which depend on the distribution of updating times that differentiates the two sticky-information models, are introduced in the next subsections.

\subsubsection{MR sticky information}

From the log-linear approximation to (3), the aggregate price level is given by:

$$
p^{M R}(t)=\int_{k \in K} f(k) p_{k}^{M R}(t) d k,
$$

with sectoral price indices evolving according to:

$$
p_{k}^{M R}(t)=\int_{-\infty}^{t} \alpha_{k} e^{-\alpha_{k}(t-s)} x_{k}^{M R}(s, t) d s .
$$

Therefore, the aggregate price level after the level shock follows:

$$
\begin{aligned}
p^{M R}(t) & =\int_{k \in K} f(k) \int_{-\infty}^{t} \alpha_{k} e^{-\alpha_{k}(t-s)} E_{s}[m(t)] d s d k \\
& =\int_{k \in K} f(k) \int_{0}^{t} \alpha_{k} e^{-\alpha_{k}(t-s)} \bar{m} e^{-\delta t} d s d k \\
& =\bar{m} e^{-\delta t} \int_{k \in K} f(k)\left(1-e^{-\alpha_{k} t}\right) d k
\end{aligned}
$$

\subsubsection{Fixed-duration sticky information}

The log-linear versions of the aggregate and sectoral price levels yield:

$$
\begin{aligned}
& p^{F D}(t)=\int_{k \in K} f(k) p_{k}^{F D}(t) d k \\
& p_{k}^{F D}(t)=\int_{t-d_{k}}^{t} \frac{1}{d_{k}} x_{k}^{F D}(s, t) d s .
\end{aligned}
$$

6 Given that I am analyzing one-time surprise shocks in an otherwise perfect foresight model, all information sets updated after $t=0$ are equal. 
Note that for $0 \leq t<\sup _{k \in K}\left\{d_{k}\right\}$ some firms still set prices based on their pre-shock plans, and that only after $t=\sup _{k \in K}\left\{d_{k}\right\}$ have all firms updated their information after the shock. Thus, the aggregate price level follows:

$$
\begin{aligned}
p^{F D}(t)= & \int_{k \in K} f(k) \int_{t-d_{k}}^{t} \frac{1}{d_{k}} E_{s}[m(t)] d s d k \\
= & \left\{\begin{array}{c}
\int_{\left\{k \in K \mid d_{k}<t\right\}} f(k) \int_{0}^{d_{k}} \frac{1}{d_{k}} \bar{m} e^{-\delta t} d s d k \quad 0 \leq t<\sup _{k \in K}\left\{d_{k}\right\} \\
+\int_{\left\{k \in K \mid d_{k} \geq t\right\}} f(k) \int_{0}^{t} \frac{1}{d_{k}} \bar{m} e^{-\delta t} d s d k
\end{array}, 0 \leq \sup _{k \in K}\left\{d_{k}\right\}\right. \\
\bar{m} e^{-\delta t}, t \geq \sup _{k \in K}\left\{d_{k}\right\} & \left\{\begin{array}{l}
\left.\bar{m} e^{-\delta t}\left(\int_{0}^{t} f(\tau) d \tau+\int_{t}^{\sup _{k \in K}\left\{d_{k}\right\}} f(\tau) \frac{t}{\tau} d \tau\right), 0 \leq t<\sup _{k}\right) \\
\bar{m} e^{-\delta t}, t \geq \sup _{k \in K}\left\{d_{k}\right\}
\end{array}\right.
\end{aligned}
$$

\subsubsection{Results}

Using the previously defined $\Gamma^{J}$ as the measure for the effects of the shock, I obtain the following result:

Proposition 3.1 Under strategic neutrality in price setting, the normalized and discounted cumulative effects of a level shock to nominal income on the output gap in the two economies are:

$$
\begin{aligned}
\Gamma^{M R} & =\int_{k \in K} f(k) \frac{1}{\alpha_{k}+\rho+\delta} d k \\
\Gamma^{F D} & =\int_{k \in K} f(k) \frac{(\delta+\rho) d_{k}-1+e^{-(\delta+\rho) d_{k}}}{(\delta+\rho)^{2} d_{k}} d k .
\end{aligned}
$$

It follows that a given shock to the level of nominal income has a larger effect, in terms of the discounted cumulative output gap, in a heterogeneous economy than in its counterfactual one-sector model:

Corollary 3.2 For a given heterogeneous economy, let $\Gamma^{J}(\bar{\alpha})$ denote the normalized and discounted cumulative effect of a level shock on the output gap in its counterfactual one-sector economy. Then:

$$
\Gamma^{J}>\Gamma^{J}(\bar{\alpha})
$$


for $J=" M R ", " F D "$.

In a limiting case of Proposition 3.1, I can relate the measure of the effects of the shock on the output gap to a moment of the distribution of durations of price plans in the heterogeneous economy:

Corollary 3.3 In the limiting case of a permanent level shock and no discounting, the following results hold:

$$
\begin{aligned}
\lim _{\delta \rightarrow 0, \rho \rightarrow 0} \Gamma^{M R} & =\int_{k \in K} f(k) \frac{1}{\alpha_{k}} d k \\
\lim _{\delta \rightarrow 0, \rho \rightarrow 0} \Gamma^{F D} & =\frac{1}{2} \int_{k \in K} f(k) d_{k} d k,
\end{aligned}
$$

so that the effects are proportional to the average duration of price plans in the economies.

These results show that the average frequency of information updating is a misleading indicator of the overall degree of informational frictions. To help understand why this is so, consider a heterogeneous economy in which a non-negligible fraction of firms collects new information continuously. Then, it will display an infinite average rate of price plan revisions, but, nevertheless, shocks can still have large effects on the output gap due to firms which only update information infrequently. Put differently, in heterogeneous economies a high average frequency of information updating needs not imply a low degree of informational frictions. Carvalho and Schwartzman (2015) show that this result can be traced back to a "selection effect" that applies to all sticky-price and sticky-information models with time-dependent pricing. They also show that, under some circumstances, the degree of monetary non-neutrality in this large class of models can be summarized by a few moments of the distribution of the duration of price plans (or, in the case of sticky-price economies, price spells).

\subsection{Growth-rate shocks}

In the case of a growth-rate shock, I assume that $\dot{m}(t) \equiv \frac{\partial m(t)}{\partial t}$ jumps at $t=0$ from $\pi$ to $\pi+\Delta \pi$, where $\Delta \pi$ is the size of the shock. Thereafter, the shock decays 
exponentially at rate $\lambda \geq 0$, so that $\dot{m}(t)=\pi+\Delta \pi e^{-\lambda t}$, and $m(t)=\int_{0}^{t} \dot{m}(s) d s=$ $\pi t+\Delta \pi \frac{1-e^{-\lambda t}}{\lambda}$. The particular case of a permanent growth-rate shock obtains when $\lambda=0$. Once again, to solve for the response of aggregate variables, the relevant distinction is between firms that already updated their pricing plans after the shock, and firms that are still setting prices based on older information. I consider each of the two models in turn.

\subsubsection{MR sticky information}

Firms which get to update their plans after $t=0$ set:

$$
\begin{aligned}
x_{k}^{M R}(s, t) & =E_{s}[m(t)] \\
& =\pi t+\Delta \pi \frac{1-e^{-\lambda t}}{\lambda},
\end{aligned}
$$

for $0 \leq s \leq t$. As a result, the aggregate price level after the shock evolves according to:

$$
\begin{aligned}
p^{M R}(t) & =\int_{k \in K} f(k) \int_{-\infty}^{t} \alpha_{k} e^{-\alpha_{k}(t-s)} E_{S}[m(t)] d s d k \\
& =\int_{k \in K} f(k) \int_{-\infty}^{0} \alpha_{k} e^{-\alpha_{k}(t-s)} \pi t d s+\int_{0}^{t} \alpha_{k} e^{-\alpha_{k}(t-s)}\left(\pi t+\Delta \pi \frac{1-e^{-\lambda t}}{\lambda}\right) d s d k \\
& =\int_{k \in K} f(k)\left(e^{-\alpha_{k} t} \pi t+\left(1-e^{-\alpha_{k} t}\right)\left(\pi t+\Delta \pi \frac{1-e^{-\lambda t}}{\lambda}\right)\right) d k \\
& =\pi t+\int_{k \in K} f(k)\left(1-e^{-\alpha_{k} t}\right) \Delta \pi \frac{1-e^{-\lambda t}}{\lambda} d k
\end{aligned}
$$

\subsubsection{Fixed-duration sticky information}

The aggregate price level is now given by:

$$
\begin{aligned}
& p^{F D}(t) \quad=\quad \int_{k \in K} f(k) \int_{t-d_{k}}^{t} \frac{1}{d_{k}} E_{s}[m(t)] d s d k \\
& =\left\{\begin{array}{l}
\int_{\left\{k \in K \mid d_{k}<t\right\}} f(k) \int_{0}^{d_{k}} \frac{1}{d_{k}}\left(\pi t+\Delta \pi \frac{1-e^{-\lambda t}}{\lambda}\right) d s d k \\
+\int_{\left\{k \in K \mid d_{k} \geq t\right\}} f(k) \int_{0}^{t} \frac{1}{d_{k}}\left(\pi t+\Delta \pi \frac{1-e^{-\lambda t}}{\lambda}\right) d s d k \quad, 0 \leq t<\sup _{k \in K}\left\{d_{k}\right\} \\
+\int_{\left\{k \in K \mid d_{k} \geq t\right\}} f(k) \int_{t}^{d_{k}} \frac{1}{d_{k}} \pi t d s d k \\
\pi t+\Delta \pi \frac{1-e^{-\lambda t}}{\lambda}, t \geq \sup _{k \in K}\left\{d_{k}\right\}
\end{array}\right. \\
& =\left\{\begin{array}{l}
\pi t+\left(\int_{0}^{t} f(\tau) d \tau+\int_{t}^{\sup _{k} \in K\left\{d_{k}\right\}} f(\tau) \frac{t}{\tau} d \tau\right) \Delta \pi \frac{1-e^{-\lambda t}}{\lambda}, 0 \leq t<\sup _{k \in K}\left\{d_{k}\right\} \\
\pi t+\Delta \pi \frac{1-e^{-\lambda t}}{\lambda}, t \geq \sup _{k \in K}\left\{d_{k}\right\}
\end{array}\right.
\end{aligned}
$$




\subsubsection{Results}

In analogy with the case of level shocks, I obtain the following results:

Proposition 3.4 Under strategic neutrality in price setting, the normalized and discounted cumulative effects of a growth rate shock to nominal income on the output gap in the two economies are:

$$
\begin{aligned}
\Gamma^{M R} & =\int_{k \in K} f(k) \frac{1}{\left(\alpha_{k}+\rho\right)\left(\alpha_{k}+\lambda+\rho\right)} d k \\
\Gamma^{F D} & =\int_{k \in K} f(k) \frac{e^{-\rho d_{k}}(\lambda+\rho)^{2}-\rho^{2} e^{-(\rho+\lambda) d_{k}}-\lambda(\lambda+2 \rho)}{d_{k} \lambda \rho^{2}(\lambda+\rho)^{2}} d k .
\end{aligned}
$$

Corollary 3.5 For a given heterogeneous economy, let $\Gamma^{J}(\bar{\alpha})$ denote the normalized and discounted cumulative effect of a growth rate shock on the output gap in its counterfactual one-sector economy. Then:

$$
\Gamma^{J}>\Gamma^{J}(\bar{\alpha})
$$

for $J=" M R ", " F D "$.

In a limiting case of Proposition 3.4, I can also relate the measure of the effects of the growth rate shock on the output gap to a moment of the distribution of durations of price plans in the heterogeneous economy:

Corollary 3.6 In the limiting case of a permanent growth rate shock and no discounting, the following results hold:

$$
\begin{aligned}
\lim _{\delta \rightarrow 0, \rho \rightarrow 0} \Gamma^{M R} & =\int_{k \in K} f(k)\left(\frac{1}{\alpha_{k}}\right)^{2} d k \\
\lim _{\delta \rightarrow 0, \rho \rightarrow 0} \Gamma^{F D} & =\frac{1}{6} \int_{k \in K} f(k) d_{k}^{2} d k
\end{aligned}
$$

so that the effects are proportional to the second moment of the distribution of (expected) durations of price plans in the economies. 


\section{Quantitative results}

This subsection presents quantitative results on the role of heterogeneity in the models under consideration. I relax some of the simplifying assumptions of Subsection 3 , and allow for strategic complementarities. To calibrate the model, I need a distribution of the frequency of price plan revisions, for which there is little information available. $^{7}$ Thus, in the absence of a clear alternative, I choose to use the statistics on the frequency of price changes in the U.S. economy reported by Bils and Klenow (2004). One possible advantage of using such data over an arbitrary distribution is that the frequency of price changes should be at least partially related to the frequency with which firms gather and process information that is relevant for their pricing decisions. ${ }^{8}$ However, because of these data limitations, the quantitative results presented next should be taken with more grains of salt than if the Bils-Klenow distribution had been used to calibrate a sticky-price model.

I associate each sector in the model with each unique frequency of adjustments among the goods and services categories listed by Bils and Klenow in the table in their appendix. ${ }^{9}$ The sectoral weights are then given by the sum of the weights for the relevant categories. This results in 142 sectors, with an average frequency of price plan revisions of once every 2.9 months, an average duration of price plans of 6.6 months, and a standard deviation of durations of such plans of 7.1 months. The (discretized versions of the) models are solved through computation of the perfect foresight responses to the one-time shocks.

\subsection{Real rigidities and quantitative results}

Recall that the analytical results derived so far rested on the simplifying assumption of no strategic complementarities/real rigidities $(\theta=1)$. Even then, the quantitative implications of accounting for heterogeneity are far from trivial. For example, for level shocks, taking the case of a permanent shock and no discounting as a bench-

7 Fabiani et al. (2005) provide some survey evidence.

8 A potentially interesting alternative would be to use a multi-sector version of the structural model of Reis (2006) to obtain the distribution of the frequency of information updating endogenously.

9 In other words, each sector aggregates all the goods and services categories that have the same frequency of adjustment. 
mark, when heterogeneity is accounted for the cumulative effects on the output gap more than double (the ratio is 6.6/2.9). For growth rate shocks, again focusing on permanent shocks and no discounting as a benchmark, the results are even more pronounced: the ratio of the cumulative effects in the heterogeneous economies to those in the one-sector economies is $\frac{(6.6)^{2}+(7.1)^{2}}{(2.9)^{2}} \approx 11.2$.

Introducing strategic complementarities in price setting exacerbates the role of heterogeneity even further. To illustrate this result I start by displaying impulse response functions (IRFs) for different shocks, for both sticky-information specifications. For growth-rate shocks, I display IRFs of output and inflation, while for level shocks I depict IRFs of output and of the aggregate price level. All comparisons involve a one-sector economy with the same average frequency of information updating.

Figures 1 and 2 display the results for the MR and fixed-duration stickyinformation models, respectively. All cases include IRFs both with and without strategic complementarities. In the economies with complementarities I set $\sigma=1$, $\varphi=0.25, \rho=0$, and $\varepsilon=\eta=8$, which yields a Ball-Romer index of real rigidities $\theta=0.15{ }^{10}$ From these results it is clear that strategic complementarities interact with heterogeneity to generate more persistent real effects of monetary shocks. Real rigidities do make the adjustment process more sluggish, even in the identical-firms case. With heterogeneity, however, this is even more so, according to several metrics: the recession troughs are delayed, output is lower than in the identical-firms economy essentially during the whole process, and takes much longer to return to the steady state; inflation is, accordingly, also more persistent. ${ }^{11}$

10 Woodford (2003, ch. 3) argues that values in the 0.10-0.15 range can be obtained with plausible assumptions for various sources of real rigidities in identical-firms models. Results with larger values of $\theta$ (less real rigidities), which imply smaller differences between heterogeneous and identical-firms economies, are presented in the next subsection.

11 Note that some responses in the fixed-duration sticky information model display kinks and "zig-zags." This is a common feature of fixed-duration price-setting models, pioneered by Taylor (1979). They occur at every point in time $t>0$ that coincides with the duration of plans of any given sector. This is more clearly understood in the case of a permanent negative level shock, say, with identical firms and no strategic complementarities. The price level will decline linearly until the time when all price plans are reset (i.e., the duration of price plans). At that point the aggregate price level reaches its new level with a non-zero derivative, and remains constant thereafter, implying a kink. This means that inflation jumps from some negative number to zero at that time. With heterogeneity, at every point in time that corresponds to the length of price plans in some sector a similar phenomenon occurs. 
Figure 1

Sticky information - Mankiw and Reis

Persistent growth rate shock: $\pi=0, \Delta \pi=-0.05, \lambda=0.14$ (half life $=5$ years)
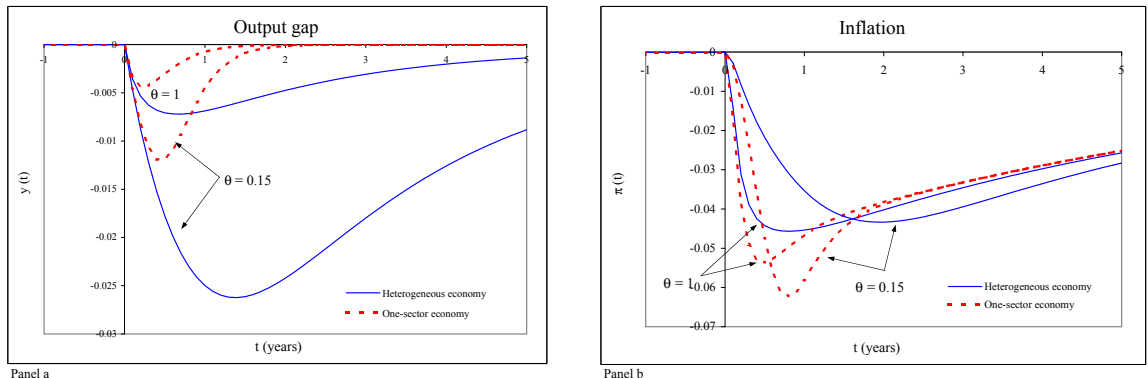

Temporary growth rate shock: $\pi=0, \Delta \pi=-0.05, \lambda=0.7$ (half life $=1$ year)
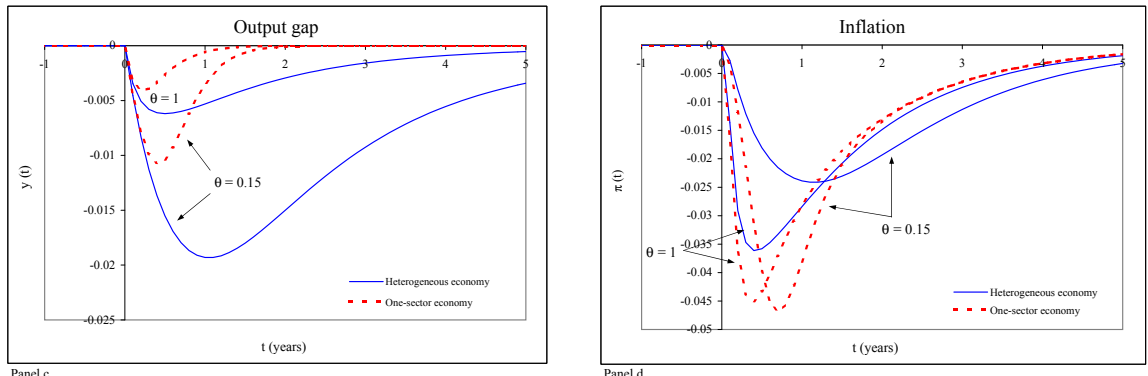

Permanent level shock: $\overline{\mathrm{m}}=-0.1, \rho=0$
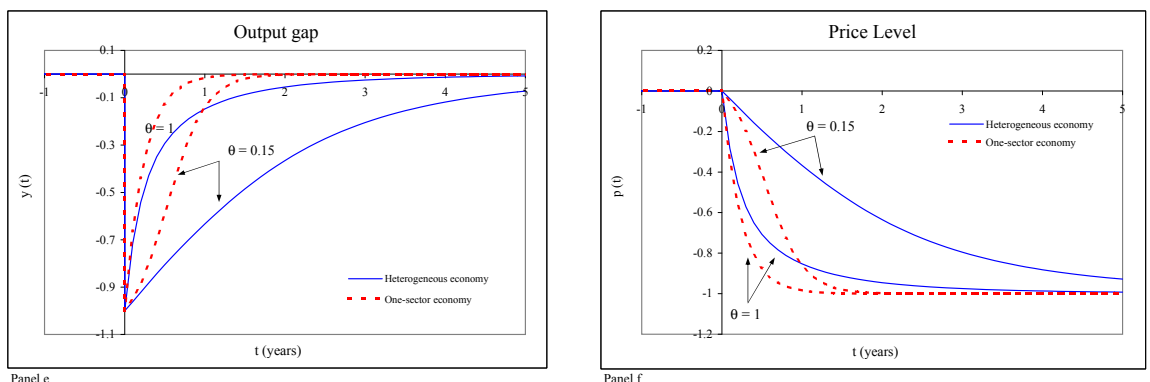
Figure 2

Sticky information - fixed duration

Persistent growth rate shock: $\pi=0, \Delta \pi=-0.05, \lambda=0.14$ (half life $=5$ years)
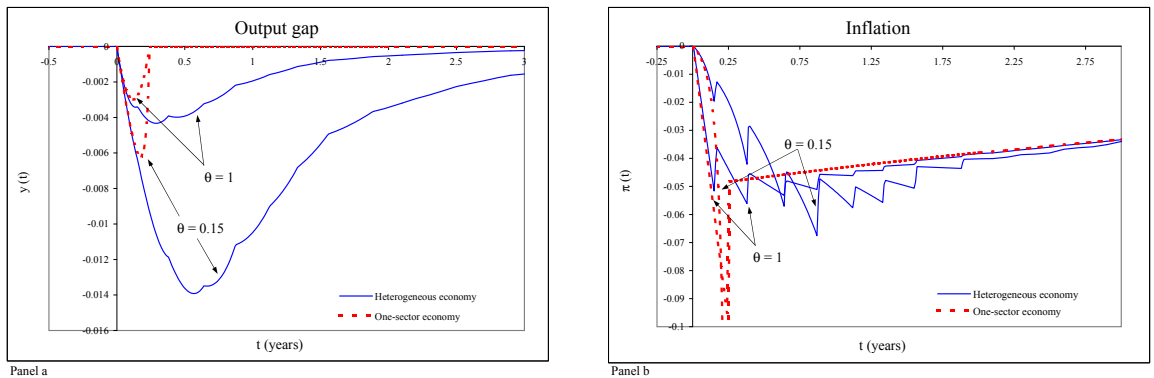

Temporary growth rate shock: $\pi=0, \Delta \pi=-0.05, \lambda=0.7$ (half life $=1$ year)
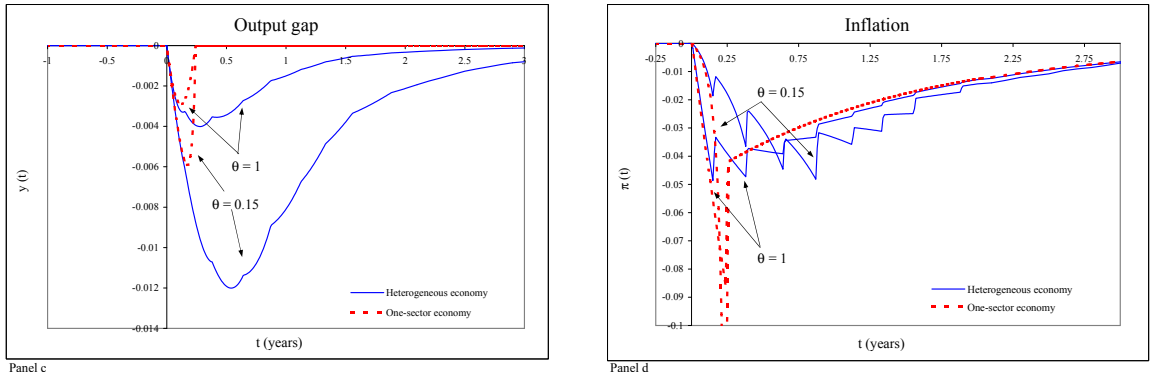

Permanent level shock: $\overline{\mathrm{m}}=-0.1, \rho=0$
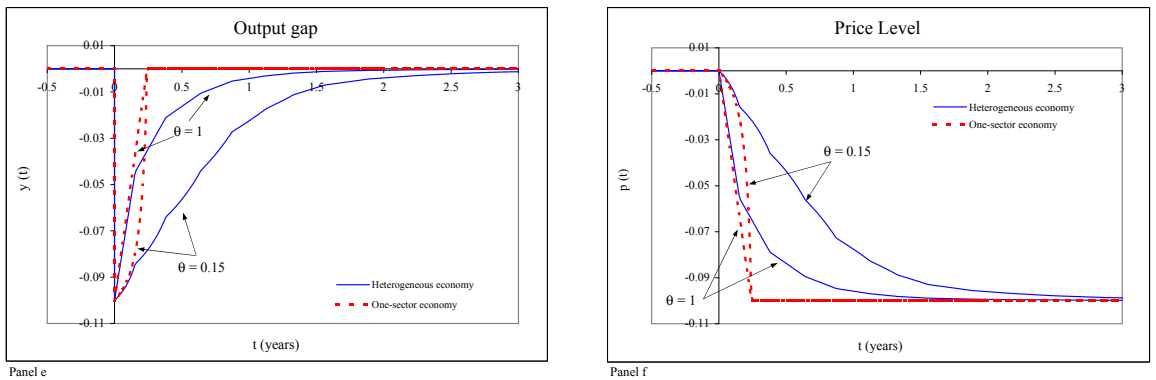
The mechanism underlying the interaction between heterogeneity and pricing complementarities is the same as that highlighted by Carvalho (2006) in the context of a sticky-price model. With no strategic complementarities, relative to an identical-firms economy with the same average frequency of price plans, heterogeneous economies initially display faster adjustment owing to a relatively higher measure of firms with higher frequency of price plan revisions, which get to update their information sets earlier. As time passes, the distribution of price plan durations among firms which have not yet updated becomes dominated by firms with relatively lower information updating rates, slowing down the adjustment process. As Carvalho and Schwartzman (2015) show, this implication of heterogeneity can be cast in terms of a selection effect.

When there are strategic complementarities, the decisions of firms in sectors in which information updating is more frequent are influenced by the existence of sectors where firms revise price plans less often, since the former do not want to set price paths that will deviate "too much" from the aggregate price in the future. ${ }^{12}$ Therefore, sectors with price plans that last longer on average end up having a disproportionate effect on the aggregate price level.

\subsection{Fitting IRFs with an identical-firms model}

In this subsection I address the question of which parameterization for an identicalfirms economy will best mimic the dynamics of a given heterogeneous economy in terms of its impulse response functions. For that purpose I perform a few related exercises. I compute the IRF of the output gap to different shocks in the heterogeneous economy, ${ }^{13}$ for different degrees of strategic complementarity. Then, I find the parameters for the identical-firms economy that minimize the integral of squared deviations of its IRF from the heterogeneous economy's IRF. In the first exercise, the identical-firms economy is constrained to have the same degree of real rigidities

The same holds for other shocks and when there are strategic complementarities, although in the latter case the kinks and zig-zags tend to be smoother.

12 On the other hand, firms in sectors in which price plans are revised less often are also influenced by the pricing decisions of firms in the relatively fast adjusting sectors, but to a lesser extent.

13 I focus the analysis on growth rate shocks, which are empirically more plausible. 
as the heterogeneous economy, and therefore I only optimize over the average duration of price plans $\left(d_{1 \mathrm{sec}}\right) .{ }^{14}$ In the second exercise I also optimize with respect to the degree of strategic complementarities in the identical-firms economy $\left(1-\theta_{1 \mathrm{sec}}\right)$.

The results are presented in Tables 1 (MR sticky information) and 2 (fixedduration sticky information). For each case, the top two sub-tables present the results when the identical-firms economy is constrained to have the same degree of strategic complementarity as the heterogeneous economy, while the bottom two report the case in which $\theta_{1 \mathrm{sec}}$ is also optimized over to provide a better fit. Given the analyses of the previous sections, one might have guessed that fitting the IRFs of a truly heterogeneous economy with an identical-firms model would require a higher average duration of price plans and perhaps more strategic complementarities than in the heterogeneous economy. This, however, is not quite the case. A higher average duration is indeed required, and the more so the higher the degree of real rigidities in the heterogeneous economy $(1-\theta)$. Despite the fact that the average duration of a price plan in the heterogeneous economy that generated the "data" is approximately 6.6 months, the "best-fitting average duration" for the identical-firms models can be as high as 11.5 months when it displays the same degree of real rigidities as the heterogeneous economy, or 31.2 months when $\theta_{1 \mathrm{sec}}$ is allowed to vary. In that case, however, the degree of strategic complementarity in the identical-firms economy is systematically lower than in the heterogeneous economy. They do vary in the same direction. These results are robust across all combinations of shocks and models.

The fit obtained in the exercise is illustrated in Figure 3 for the MR sticky information case. It displays the IRFs (for output gap and inflation) for the heterogeneous economy for a permanent growth rate shock, as well as three identical-firms economies: one with the same average frequency of price plans revisions and degree of real rigidities as the heterogeneous economy ("No fit"), and the two best-fitting identical-firms economies corresponding to the two exercises ("Best fit $d, \theta$ " and "Best fit $d ")$. The reason why the best fit is obtained by lowering the average frequency of price plan revisions and reducing the degree of strategic complementarities (often to the extent of implying strategic substitutability $-\theta_{1 \mathrm{sec}}>1-$ rather than complementarity) is clear from the picture. When $\theta_{1 \text { sec }}$ is constrained to be equal

14 The "1 sec" subscript stands for "one sector" (i.e., the model with identical firms). 
Figure 3

Best fit: sticky information - Mankiw and Reis

Permanent Growth Rate Shock: $\pi=0.05, \Delta \pi=-0.05, \lambda=0$

"No fit": $\mathrm{d}_{1 \mathrm{sec}}=2.9$ months, $\theta_{1 \mathrm{sec}}=0.15$

"Best fit d": $d_{1 \mathrm{sec}}=9.5$ months, $\theta_{1 \mathrm{sec}}=0.15$

"Best fit d, $\theta$ ": $\quad d_{1 \mathrm{sec}}=25$ months, $\theta_{1 \mathrm{sec}}=1.74$

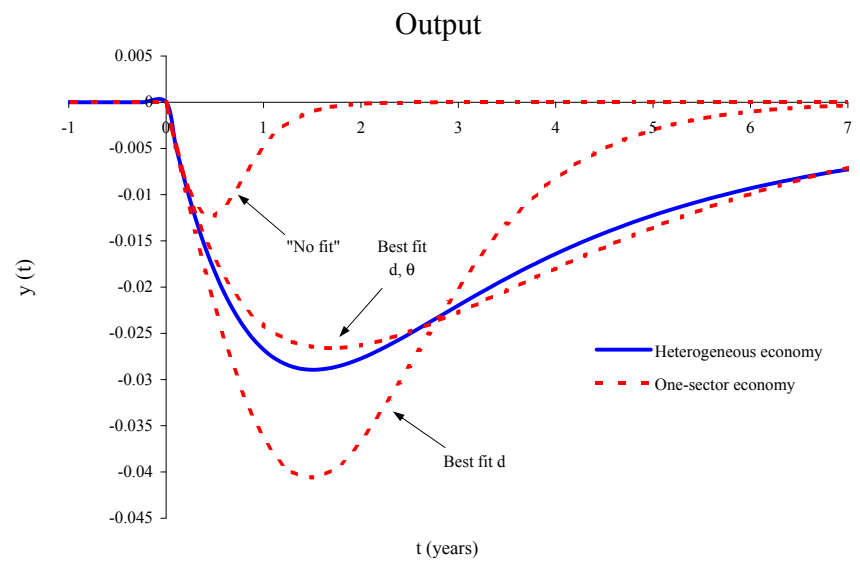

Panel a

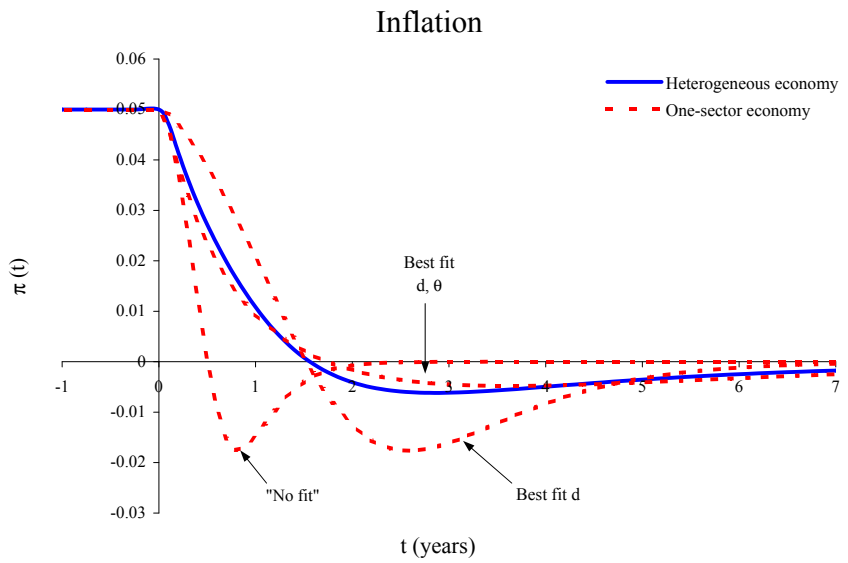

Panel b 
Table 1

Fitting one-sector economy - Mankiw and Reis

\begin{tabular}{|c|c|c|c|c|c|}
\hline \multicolumn{6}{|c|}{ Optimizing over $d_{1 \mathrm{sec}}$, setting $\theta_{1 \mathrm{sec}}=\theta$} \\
\hline & \multicolumn{2}{|c|}{$\lambda=0$} & \multicolumn{2}{|c|}{$\lambda=0.5$} & \\
\hline & $\theta$ & $\mathrm{d}_{1 \mathrm{sec}}$ & $\theta$ & $\mathrm{d}_{1 \mathrm{sec}}$ & \\
\hline & 0.10 & 10.0 & 0.10 & 9.3 & \\
\hline & 0.15 & 9.5 & 0.15 & 8.7 & \\
\hline & 0.25 & 8.8 & 0.25 & 8.1 & \\
\hline & 0.50 & 8.0 & 0.50 & 7.4 & \\
\hline & 1.00 & 7.4 & 1.00 & 6.8 & \\
\hline \multicolumn{6}{|c|}{ Optimizing over both $d_{1 \mathrm{sec}}$ and $\theta_{1 \mathrm{sec}}$} \\
\hline \multicolumn{3}{|c|}{$\lambda=0$} & \multicolumn{3}{|c|}{$\lambda=0.5$} \\
\hline$\theta$ & $\mathrm{d}_{1 \mathrm{sec}}$ & $\theta_{1 \mathrm{sec}}$ & $\theta$ & $\mathrm{d}_{1 \mathrm{sec}}$ & $\theta_{1 \mathrm{sec}}$ \\
\hline 0.10 & 26.5 & 1.32 & 0.10 & 22.1 & 0.90 \\
\hline 0.15 & 25.2 & 1.74 & 0.15 & 21.2 & 1.24 \\
\hline 0.25 & 24.4 & 2.15 & 0.25 & 20.8 & 1.57 \\
\hline 0.50 & 22.6 & 4.51 & 0.50 & 19.7 & 3.54 \\
\hline 1.00 & 21.7 & 8.29 & 1.00 & 19.3 & 6.80 \\
\hline
\end{tabular}

Table 2

Fitting one-sector economy - fixed duration

\begin{tabular}{|c|c|c|c|c|c|}
\hline \multicolumn{6}{|c|}{ Optimizing over $d_{1 \mathrm{sec}}$, setting $\theta_{1 \mathrm{sec}}=\theta$} \\
\hline & \multicolumn{2}{|c|}{$\lambda=0$} & \multicolumn{2}{|c|}{$\lambda=0.5$} & \\
\hline & $\theta$ & $\mathrm{d}_{1 \mathrm{sec}}$ & $\theta$ & $\mathrm{d}_{1 \mathrm{sec}}$ & \\
\hline & 0.10 & 11.5 & 0.10 & 11.3 & \\
\hline & 0.15 & 10.4 & 0.15 & 10.2 & \\
\hline & 0.25 & 9.2 & 0.25 & 9.1 & \\
\hline & 0.50 & 7.8 & 0.50 & 7.6 & \\
\hline & 1.00 & 6.6 & 1.00 & 6.5 & \\
\hline \multicolumn{6}{|c|}{ Optimizing over both $d_{1 \mathrm{sec}}$ and $\theta_{1 \mathrm{sec}}$} \\
\hline \multicolumn{3}{|c|}{$\lambda=0$} & \multicolumn{3}{|c|}{$\lambda=0.5$} \\
\hline$\theta$ & $\mathrm{d}_{1 \mathrm{sec}}$ & $\theta_{1 \mathrm{sec}}$ & $\theta$ & $\mathrm{d}_{1 \mathrm{sec}}$ & $\theta_{1 \mathrm{sec}}$ \\
\hline 0.10 & 31.2 & 4.05 & 0.10 & 27.9 & 3.03 \\
\hline 0.15 & 28.4 & 4.54 & 0.15 & 25.3 & 3.53 \\
\hline 0.25 & 26.0 & 5.00 & 0.25 & 23.2 & 4.60 \\
\hline 0.50 & 22.3 & 8.35 & 0.50 & 21.1 & 7.32 \\
\hline 1.00 & 20.8 & 13.94 & 1.00 & 19.8 & 12.56 \\
\hline
\end{tabular}

to $\theta$, the decrease in the average frequency of information updating tends to produce effects that are larger and less persistent than in the heterogeneous economy. 
Increasing both $d_{1 \text { sec }}$ and $\theta_{1 \text { sec }}$ attenuates the magnitude of the effects of the shock and increases its persistence, therefore providing a better fit.

\section{Conclusion}

In this paper, I explore the implications of ex-ante heterogeneity in the degree of information stickiness for aggregate dynamics. The reslts show that the effects of heterogeneity in price-setting behavior first documented in the context of sticky-price models (Carvalho 2006) carry over to two sticky-information models. I introduce heterogeneity in the degree of information stickiness across sectors into otherwise standard models, and present both qualitative and quantitative results showing that heterogeneity in information stickiness leads various shocks to have larger and more persistent effects on the output gap than in the corresponding counterfactual onesector economy. In particular, reproducing the dynamics of a heterogeneous stickyinformation economy with an identical-firms model requires calibrating the average frequency of information updating to be lower than the natural calibration that matches the average frequency of information updating in the original heterogeneous economy.

\section{References}

Ball, L. and D. Romer (1990), Real Rigidities and the Non-Neutrality of Money, Review of Economic Studies 57: 183-203.

Bils, M. and P. Klenow (2004), Some Evidence on the Importance of Sticky Prices, Journal of Political Economy 112: 947-985 (also NBER Working Paper \#9069).

Carvalho, C. (2006), Heterogeneity in Price Stickiness and the Real Effects of Monetary Shocks, Frontiers of Macroeconomics: Vol. 2 : Iss. 1, Article 1.

Carvalho, C. and F. Schwartzman (2015), Selection and Monetary Non-neutrality in Time-dependent Pricing Models, Journal of Monetary Economics 76: 141-156.

Dupor, B. and T. Tsuruga (2005), Sticky Information: The Impact of Different Information Updating Assumptions, Journal of Money, Credit and Banking 37: $1143: 1152$. 
Fabiani, S., M. Druant, I. Hernando, C. Kwapil, B. Landau, C. Loupias, F. Martins, T. Mathä, R. Sabbatini, H. Stahl, A. Stokman (2005), The Pricing Behaviour of Firms in the Euro Area: New Survey Evidence, Banco de España Working Papers 536.

Mankiw, G., and R. Reis (2002), Sticky Information Versus Sticky Prices: A Proposal to Replace the New Keynesian Phillips Curve, Quarterly Journal of Economics 117: 1295-1328.

Mankiw, G., and R. Reis (2006), Pervasive Stickiness, American Economic Review, Papers and Proceedings, 96: 164-169.

Mankiw, G., and R. Reis (2007), Sticky Information in General Equilibrium, Journal of the European Economic Association 5: 603-613.

Reis, R. (2006), Inattentive Producers, Review of Economic Studies 73: 793-821.

Taylor, J. (1979), Staggered Wage Setting in a Macro Model, American Economic Review 69: 108-113.

Woodford, M. (2003), Interest and Prices: Foundations of a Theory of Monetary Policy, Princeton University Press. 


\section{A. Appendix}

\section{A.1 Proofs of propositions}

Proposition 3.1 Under strategic neutrality in price setting, the normalized and discounted cumulative effects of a level shock to nominal income on the output gap in the two economies are:

$$
\begin{aligned}
\Gamma^{M R} & =\int_{k \in K} f(k) \frac{1}{\alpha_{k}+\rho+\delta} d k, \\
\Gamma^{F D} & =\int_{k \in K} f(k) \frac{(\delta+\rho) d_{k}-1+e^{-(\delta+\rho) d_{k}}}{(\delta+\rho)^{2} d_{k}} d k .
\end{aligned}
$$

Proof For the MR sticky-information model:

$$
\begin{aligned}
\Gamma^{M R} & =\frac{1}{\bar{m}} \int_{0}^{\infty} e^{-\rho t}\left(y^{M R}(t)-y^{n}(t)\right) d t \\
& =\frac{1}{\bar{m}} \int_{0}^{\infty} e^{-\rho t}\left(m(t)-p^{M R}(t)\right) d t \\
& =\frac{1}{\bar{m}} \int_{0}^{\infty} e^{-\rho t}\left(\bar{m} e^{-\delta t}-\bar{m} e^{-\delta t} \int_{k \in K} f(k)\left(1-e^{-\alpha_{k} t}\right) d k\right) d t \\
& =\int_{0}^{\infty} \int_{k \in K} f(k)\left[e^{-(\delta+\rho) t}-e^{-(\delta+\rho) t}\left(1-e^{-\alpha_{k} t}\right)\right] d k d t \\
& =\int_{k \in K} f(k) \int_{0}^{\infty} e^{-(\delta+\rho) t} e^{-\alpha_{k} t} d t d k \\
& =\int_{k \in K} f(k) \frac{1}{\alpha_{k}+\rho+\delta} d k .
\end{aligned}
$$

For the fixed duration sticky-information model:

$$
\begin{aligned}
& \Gamma^{F D}=\frac{1}{\bar{m}} \int_{0}^{\infty} e^{-\rho t}\left(y^{F D}(t)-y^{n}(t)\right) d t \\
& =\frac{1}{\bar{m}} \int_{0}^{\infty} e^{-\rho t}\left(m(t)-p^{F D}(t)\right) d t \\
& =\frac{1}{\bar{m}} \int_{0}^{\sup _{k \in K}\left\{d_{k}\right\}} e^{-\rho t}\left(\bar{m} e^{-\rho t}-\bar{m} e^{-\rho t}\left(\int_{0}^{t} f(\tau) d \tau+\int_{t}^{\sup _{k \in K}\left\{d_{k}\right\}} f(\tau) \frac{t}{\tau} d \tau\right)\right) d t \\
& =\int_{0}^{\sup _{k \in K}\left\{d_{k}\right\}} e^{-(\delta+\rho) t}-e^{-(\delta+\rho) t}\left(\int_{0}^{t} f(\tau) d \tau+\int_{t}^{\sup _{k} \in K\left\{d_{k}\right\}} f(\tau) \frac{t}{\tau} d \tau\right) d t
\end{aligned}
$$

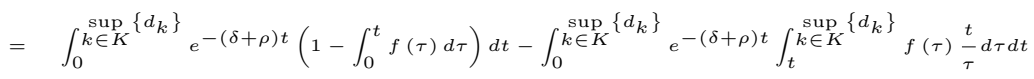

$$
\begin{aligned}
& =\left[-(1-F(t)) \frac{e^{-(\delta+\rho) t}}{\delta+\rho}\right]_{t=0}^{\sup _{k \in K}\left\{d_{k}\right\}}-\int_{0} \sup _{k \in K}\left\{d_{k}\right\} \frac{e^{-(\delta+\rho) \tau}}{\delta+\rho} f(\tau) d \tau
\end{aligned}
$$




$$
\begin{aligned}
& -\left(\begin{array}{c}
{\left[\int_{t}^{\sup _{k \in K}\left\{d_{k}\right\}} \frac{f(\tau)}{\tau} d \tau\left(-\frac{1}{(\delta+\rho)^{2}}-\frac{t}{\delta+\rho}\right) e^{-(\delta+\rho) t}\right]_{t=0}^{\sup _{k \in K}\left\{d_{k}\right\}}} \\
-\int_{0}^{k \in K}\left\{d_{k}\right\} \\
\left(\frac{1}{(\delta+\rho)^{2}}+\frac{\tau}{\delta+\rho}\right) e^{-(\delta+\rho) \tau} \frac{f(\tau)}{\tau} d \tau
\end{array}\right) \\
& =\frac{1}{\delta+\rho}-\int_{0}^{\sup _{k \in K}\left\{d_{k}\right\}} \frac{e^{-(\delta+\rho) \tau}}{\delta+\rho} f(\tau) d \tau-\frac{1}{(\delta+\rho)^{2}} \int_{0}^{\sup _{k \in K}\left\{d_{k}\right\}} f(\tau) \frac{1}{\tau} d \tau \\
& +\int_{0}^{\sup _{k \in K}\left\{d_{k}\right\}}\left(\frac{1}{(\delta+\rho)^{2}} \frac{1}{\tau}+\frac{1}{\delta+\rho}\right) e^{-(\delta+\rho) \tau} f(\tau) d \tau \\
& =\frac{1}{\delta+\rho}\left(1-\int_{0}^{\sup _{k \in K}\left\{d_{k}\right\}} \frac{1}{\delta+\rho} f(\tau) \frac{1}{\tau} d \tau+\int_{0}^{\sup _{k \in K}\left\{d_{k}\right\}} \frac{1}{\delta+\rho} \frac{1}{\tau} e^{-(\delta+\rho) \tau} f(\tau) d \tau\right) \\
& =\frac{1}{\delta+\rho}\left(1-\int_{0}^{\sup _{k \in K}\left\{d_{k}\right\}} f(\tau) \frac{1-e^{-(\delta+\rho) \tau}}{(\delta+\rho) \tau} d \tau\right) \\
& =\int_{0}^{\sup _{k \in K}\left\{d_{k}\right\}} f(\tau) \frac{(\delta+\rho) \tau-1+e^{-(\delta+\rho) \tau}}{(\delta+\rho)^{2} \tau} d \tau=\int_{k \in K} f(k) \frac{(\delta+\rho) d_{k}-1+e^{-(\delta+\rho) d_{k}}}{(\delta+\rho)^{2} d_{k}} d k .
\end{aligned}
$$

Corollary 3.2 For a given heterogeneous economy, let $\Gamma^{J}(\bar{\alpha})$ denote the normalized and discounted cumulative effect of a level shock on the output gap in its counterfactual one-sector economy. Then:

$$
\Gamma^{J}>\Gamma^{J}(\bar{\alpha})
$$

for $J=$ "MR", " $F D "$.

Proof The proof follows directly from the facts that $\frac{1}{\alpha_{k}+\rho+\delta}$ is convex in $\alpha_{k}$, and $\frac{(\delta+\rho) d_{k}-1+e^{-(\delta+\rho) d_{k}}}{(\delta+\rho)^{2} d_{k}}$ is convex in $d_{k}^{-1}$.

Corollary 3.3 In the limiting case of a permanent level shock and no discounting, the following results hold:

$$
\begin{aligned}
\lim _{\delta \rightarrow 0, \rho \rightarrow 0} \Gamma^{M R} & =\int_{k \in K} f(k) \frac{1}{\alpha_{k}} d k, \\
\lim _{\delta \rightarrow 0, \rho \rightarrow 0} \Gamma^{F D} & =\frac{1}{2} \int_{k \in K} f(k) d_{k} d k,
\end{aligned}
$$

so that the effects are proportional to the average duration of price plans in the economies. 
Proof The proof follows directly from taking limits in the expressions from Proposition 3.1:

$$
\begin{aligned}
& \lim _{\delta \rightarrow 0, \rho \rightarrow 0} \int_{k \in K} f(k) \frac{1}{\alpha_{k}+\rho+\delta} d k \\
& \lim _{\delta \rightarrow 0, \rho \rightarrow 0} \int_{k \in K} f(k) \frac{(\delta+\rho) d_{k}-1+e^{-(\delta+\rho) d_{k}}}{(\delta+\rho)^{2} d_{k}} d k .
\end{aligned}
$$

Proposition 3.4 Under strategic neutrality in price setting, the normalized and discounted cumulative effects of a growth rate shock to nominal income on the output gap in the two economies are:

$$
\begin{aligned}
\Gamma^{M R} & =\int_{k \in K} f(k) \frac{1}{\left(\alpha_{k}+\rho\right)\left(\alpha_{k}+\lambda+\rho\right)} d k \\
\Gamma^{F D} & =\int_{k \in K} f(k) \frac{e^{-\rho d_{k}}(\lambda+\rho)^{2}-\rho^{2} e^{-(\rho+\lambda) d_{k}}-\lambda(\lambda+2 \rho)}{d_{k} \lambda \rho^{2}(\lambda+\rho)^{2}} d k .
\end{aligned}
$$

Proof For the MR sticky-information model:

$$
\begin{aligned}
\Gamma^{M R} & =\frac{1}{\Delta \pi} \int_{0}^{\infty} e^{-\rho t}\left(y^{M R}(t)-y^{n}(t)\right) d t \\
& =\frac{1}{\Delta \pi} \int_{0}^{\infty} e^{-\rho t}\left(m(t)-p^{M R}(t)\right) d t \\
& =\int_{0}^{\infty} e^{-\rho t} \frac{1-e^{-\lambda t}}{\lambda}\left(1-\int_{k \in K} f(k)\left(1-e^{-\alpha_{k} t}\right) d k\right) d t \\
& =\int_{0}^{\infty} \int_{k \in K} f(k) \frac{1-e^{-\lambda t}}{\lambda} e^{-\rho t} e^{-\alpha_{k} t} d k d t \\
& =\int_{k \in K} f(k) \int_{0}^{\infty} \frac{1-e^{-\lambda t}}{\lambda} e^{-\rho t} e^{-\alpha_{k} t} d t d k \\
& =\int_{k \in K} f(k) \frac{1}{\left(\alpha_{k}+\rho\right)\left(\alpha_{k}+\lambda+\rho\right)} d k .
\end{aligned}
$$


For the fixed duration sticky-information model:

$$
\begin{aligned}
& \Gamma^{F D}=\frac{1}{\Delta \pi} \int_{0}^{\infty} e^{-\rho t}\left(y^{F D}(t)-y^{n}(t)\right) d t \\
& =\frac{1}{\Delta \pi} \int_{0}^{\infty} e^{-\rho t}\left(m(t)-p^{F D}(t)\right) d t \\
& =\int_{0}^{\sup _{k \in K}\left\{d_{k}\right\}} e^{-\rho t}\left[\frac{1-e^{-\lambda t}}{\lambda}-\left(\int_{0}^{t} f(\tau) d \tau+\int_{t}^{\sup _{k \in K}\left\{d_{k}\right\}} f(\tau) \frac{t}{\tau} d \tau\right) \frac{1-e^{-\lambda t}}{\lambda}\right] d t
\end{aligned}
$$

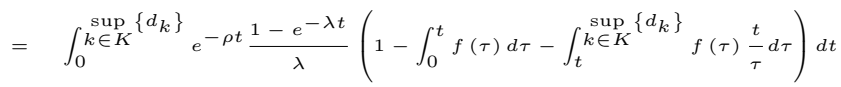

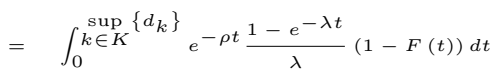

$$
\begin{aligned}
& -\int_{0}^{\sup _{k \in K}\left\{d_{k}\right\}} e^{-\rho t} \frac{1-e^{-\lambda t}}{\lambda} t\left(\int_{t}^{\sup _{k}\left\{d_{k}\right\}} f(\tau) \frac{1}{\tau} d \tau\right) d t \\
& =\left(\frac{1}{\lambda \rho}-\frac{1}{\lambda(\rho+\lambda)}\right)+\int_{0} \sup _{k \in K}\left\{d_{k}\right\} f(t)\left(\frac{e^{-(\rho+\lambda) t}}{\lambda(\rho+\lambda)}-\frac{e^{-\rho t}}{\lambda \rho}\right) d t \\
& -\left(\int_{0} \sup _{k \in K}\left\{d_{k}\right\} f(t) \frac{1}{t} d t\right) \frac{\lambda+2 \rho}{\rho^{2}(\lambda+\rho)^{2}} \\
& -\int_{0} \sup _{k \in K}\left\{d_{k}\right\} f(t) \frac{e^{-(\rho+\lambda) t}\left(e^{-\lambda t}(\lambda+\rho)^{2}(1+\rho t)+\rho^{2}(1+(\rho+\lambda) t)\right)}{t \lambda \rho^{2}(\lambda+\rho)^{2}} d t \\
& =\int_{k \in K} f(k) \frac{e^{-\rho d_{k}(\lambda+\rho)^{2}-\rho^{2} e^{-(\rho+\lambda) d_{k}}-\lambda(\lambda+2 \rho)}}{d_{k} \lambda \rho^{2}(\lambda+\rho)^{2}} d k .
\end{aligned}
$$

Corollary 3.5 For a given heterogeneous economy, let $\Gamma^{J}(\bar{\alpha})$ denote the normalized and discounted cumulative effect of a level shock on the output gap in its counterfactual one-sector economy. Then:

$$
\Gamma^{J}>\Gamma^{J}(\bar{\alpha})
$$

for $J=" M R ", " F D "$.

Proof The proof follows directly from the facts that $\frac{1}{\left(\alpha_{k}+\rho\right)\left(\alpha_{k}+\lambda+\rho\right)}$ is convex in $\alpha_{k}$, and $\frac{e^{-\rho d_{k}}(\lambda+\rho)^{2}-\rho^{2} e^{-(\rho+\lambda) d_{k}}-\lambda(\lambda+2 \rho)}{d_{k} \lambda \rho^{2}(\lambda+\rho)^{2}}$ is convex in $d_{k}^{-1}$. 
Corollary 3.6 In the limiting case of a permanent level shock and no discounting, the following results hold:

$$
\begin{aligned}
\lim _{\delta \rightarrow 0, \rho \rightarrow 0} \Gamma^{M R} & =\int_{k \in K} f(k)\left(\frac{1}{\alpha_{k}}\right)^{2} d k \\
\lim _{\delta \rightarrow 0, \rho \rightarrow 0} \Gamma^{F D} & =\frac{1}{6} \int_{k \in K} f(k) d_{k}^{2} d k
\end{aligned}
$$

so that the effects are proportional to the average duration of price plans in the economies.

Proof The proof follows directly from taking limits in the expressions from Proposition 3.4:

$$
\begin{aligned}
& \lim _{\delta \rightarrow 0, \rho \rightarrow 0} \int_{k \in K} f(k) \frac{1}{\left(\alpha_{k}+\rho\right)\left(\alpha_{k}+\lambda+\rho\right)} d k, \\
& \lim _{\delta \rightarrow 0, \rho \rightarrow 0} \int_{k \in K} f(k) \frac{e^{-\rho d_{k}}(\lambda+\rho)^{2}-\rho^{2} e^{-(\rho+\lambda) d_{k}}-\lambda(\lambda+2 \rho)}{d_{k} \lambda \rho^{2}(\lambda+\rho)^{2}} d k .
\end{aligned}
$$

